\title{
Stato attuale delle conoscenze sugli Histeridae ipogei*
}

di

\author{
Vincenzo VOMERO**
}

L'ordine dei Colectteri è quello che presenta senza dubbio il maggior numero di specie che hanno colonizzato l'ambiente ipogeo ed in modo particolare quello di grotta. L'adattamento a questo ambiente "limite" si riscontra esclusivamente in alcune famiglie in un certo modo preadattate alla vita in un ambiente ad alto tenore di umidità, dove la mancanza di luce e la temperatura pressochè costante condizionano in misura notevole l'insediamento di popolazioni in grado di riprodursi. Le famiglie che con maggior successo hanno colonizzato l'ambiente cavernicolo sono quelle dei Carabidae e dei Catopidae ed in misura notevolmente minore quelle degli Pselaphidae, Staphylinidae ed Histeridae.

Uno dei motivi principali per cui gli Histeridae sono così scarsamente presenti in ambienti ipogei è da ricercarsi nel fatto che ci troviamo di fronte ad animali già notevolmente specializzati e per regime dietetico e per esigenze ambientali. Gli adulti, come le larve, sono in massima parte carnivori, molti vivono in carogne, escrementi e materiali vegetali in decomposizione dove predano gli innumerevoli insetti associati a tali ambiente, per lo più larve di Ditteri; alcuni vivono in nidi di uccelli ed in tane di mammiferi; altri si trovano comunemente sotto cortecce di alberi morenti; in gran numero, specialmente ai tropici, sono mirmecofili e termitofili, ad un notevole grado di sinfilia; non rari sono i casi di predazione specifica (larve di alcuni Chrysomelidae e di alcuni Lepidotteri eteroceri); pochi sono humicoli ed è probabile che alcuni di questi ultimi possano nutrirsi di ife fungine come ad esempio alcune specie di Epierus e di Aeletes.

Tra le sei sottofamiglie nelle quali è divisa attualmente la famiglia Histeridae, soltanto due presentano generi che mostrano una certa elettività per l'ambiente humicolo: Abraeinae e Dendrophilinae; ed è appunto nell'ambito di queste due sottofamiglie che si possono includere gli Histeridae troglobi ed edafobi.

E'necessario ricordare che fino a non molto tempo fa queste forme specializzate erano incluse tutte in Abraeinae; uno studio più accurato però ha mostrato che Abraeinae (intesa nel senso dei vecchi autori) era una sottofamiglia altamente polifiletica; Wenzel (1944) allontanò dagli Abraeinae alcuni generi come Bacanius Lec. Anapleus, Horn, e Abraeomorphus Reitt. ponendoli in Dendrophilinae; successivamente (Vomero 1973) anche Sardulus Patrizi è stato spostato da Abraeinae a Dendrophilinae. Non è improbabile che anche questa sistemazione sia innaturale, ed è appunto per chiarire questo problema che ho iniziato una revisione

\footnotetext{
* Comunicazione presentata al Simposio sugli "Artropodi di caverna" nel IX Congresso Italiano di Entomologia, Siena 21-25 giugno 1972.

** Istituto di Zoologia di Roma, Viale dell'Università, 32 - 00100 ROMA.
} 
di Bacanius e dei generi ad esso affini che per comodità verrano chiamati "gruppo Bacanius".

Gli Histeridae in genere presentano rare specie troglobie e le poche descritte prima della scoperta di Troglobacanius Vomero appartengono tutte alla fauna paleartica. Gli Histeridae troglobi paleartici sono però rari e molto localizzati, mentre le quattro specie di Troglobacanius in Messico presentano una distribuzione relativamente ampia. Generalizzando si può ipotizzare che questa famiglia, a massima diffusione tropicale, sia rappresentata nelle grotte dell'America tropicale proporzionalmente più di altre famiglie quali appunto Carabidae e Catopidae, che nelle grotte paleartiche sono presenti in numero notevole. Le grotte temperate della regione paleartica pur essendo state studiate ad un buon livello per quanto riguarda il popolamento animale, hanno portato alla scoperta di pochissimi esempi di Histeridae troglobi; solo Speleacritus anophthalmus Jeannel può considerarsi veramente troglobio. Per quanto riguarda le grotte tropicali, due soli gruppi di ricerche biospeleologiche in Messico e cioè quelle americane e le due italiane hanno portato la conoscenza del popolamento isteridologico di quelle grotte ad un buono livello: un genere troglobio con quattro specie ampiamente distribuite nelle grotte messicane e numerosi generi troglofili e guanobi.

Scopo di questa comunicazione è una messa a punto delle attuali conoscenze sugli Histeridae ipogei con particolare riguardo a quelli cavernicoli. Dato l'esiguo numero di specie converra trattare di ognuna separatamente.

\section{Sottofam. Abraeinae}

\section{Speleacritus anophtalmus Jeannel 1934}

Primo Histeridae troglobio descritto nel 1934 su un unico esemplare, rimasto tale, proveniente dalla grotta di Fersine, a sud del lago Beysehir in Asia minore.

Gli occhi sono completamente assenti e le zampe presentano un allungamento notevole relativamente alla facies generale della famiglia. La depigmentazione del tegumento non è certamente dovuta all'effetto dell'ambiente cavernicolo, infatti alcune specie di Acritus e di altri generi humicoli sono depigmentati allo stesso modo. Resta poi da considerare quanto scrive Jeannel (1934) a proposito di modificazioni che hanno portato l'Histeridae a perdere l'uso di alcune attitudini di difesa. Nella quasi totalità degli Histeridae infatti la testa è retraibile per gran parte nel protorace dove, inflettendosi leggermente, viene a nascondere i pezzi boccali contro il prosterno; in Speleacritus invece, secondo Jeannel, la testa, con la parte anterire molto allungata, ha perduto ogni possibilità di ritrarsi contro la "mentonniere" del prosterno.

Jeannel, in base alla sola morfologia esterna di un unico esemplare, pone Spelaeacritus nella sottofamiglia Abraeinae vicino ad Acritus; e continua formulando ipotesi paleogeografiche in base a queste presunte affinità. Sarebbe notevolmente interessante poter studiare un esemplare di sesso maschile alla luce delle più moderne concezioni sulla sistematica della sottofamiglia. 
Spelaeabraeus agazzii Moro 1957 e S. cavallensis Rossi 1967

Descritto della grotta denominata "Busa delle fave" presso Refrontolo (Treviso) $S$. agazzii è conosciuto anche di una grotta del Montello di dove è stata descritta la ssp. infidus Busulini; sul M. Cavallo, dalla valle del F. Meschio alla val Cellina sono presenti altre popolazioni considerate da Rossi distinte specificamente da $S$. agazzii e descritte col nome di $S$. cavallensis. Rossi è propenso a considerare anche la ssp. infidus come specie buona. S. cavallensis è stato raccolto esclusivamente mediante l'uso delle bottiglie - trappole per endogei (vedi Rossi 196); sembra popolare la rete di microfessure presenti nelle rocce calcaree insieme ad una ricca biocenosi di "endogei".

Tutti gli Spelaeabraeus presentano anoftalmia completa e modesto sviluppo delle appendici, sono atteri e presentano un notevole grado di saldatura delle elitre.

Le due specie, che differiscono poco da un punto di vista morfologico, sembrano essere legate l'una all'ambiente cavernicolo e l'altra al così detto ambiente freatico terrestre; è probabile tuttavia che si tratti di un insieme di popolazioni forse conspecifiche comunemente presenti in una vasta zona delle Prealpi Carniche occidentali e popolanti le fessurazioni delle rocce impenetrabili all'uomo, mediante le quali possono avere accesso ad un gran numero di grotte presenti in quella regione.

Sarebbe opportuna una ricerca approfondita e metodica in tutte le cavità della zona onde accertarsi delle esigenze ambientali delle varie popolazioni. E' anche in preparazione una revisione del genere.

\section{Sottofam. Dendrophilinae}

Sardulus spelaeus Patrizi 1955

Descritto della grotta di Toddeitto, nel territorio di Dorgali in Sardegna, il genere presenta notevoli affinità con Bacanius ed Abraeomorphus.

Oltre alla totale atrofia degli occhi, all'atterismo ed alla fusione delle elitre, non sembra aver subitc altre modifiche strutturali. Il suo tenore di vita sembra essere saproxilobio, infatti è stato trovato in un pezzo di ginepro molto umido e completamente putrefatto; il ritrovamento di una pupa nello stesso pezzo di legno indica certamente un optimum ambientale trovato dall'Histeridae nelle sale di questa grotta che sembra abbondare di pezzi di legno introdotti dell'esterno.

\section{Bacanius acuminatus Casey 1893}

Fu descritto nel 1893 su esemplari provenienti dalla California (U.S.A.) e da allora non mi risultano successive segnalazioni di questo interessante Histeridae cieco e attero che in base alla morfologia esterna sembra essere un Bacanius sensu stricto.

Ho in studio un esemplare di questa rarissima e interessantissima specie, comunicatami insieme ad altro interessante materiale dal Dr. R.L. Wenzel che qui sentitamente ringrazio, che proviene dalle Oakland Hills in California.

Molto poco si conosce sull'ecologia di questa specie; dell'esemplare californiano Wenzel (com. pers.) dice che "lives in deep duff of the forest floor". 


\section{Troglobacanius Vomero 1973}

Tra il materiale riportato dalla seconda spedizione biospeleologica dell'Istituto di Zoologia dell'Università di Roma in Messico è presente un discreto numero di Histeridae provenienti per lo più da grotte tropicali; per la massima parte si tratta di troglofili (guanobi). Un unico esemplare, però, risultato appartenere ad un nuovo genere, Troglobacanius Vomero (1973), presenta caratteri specializzati alla vita cavernicola. Questa specie descritta col nome di $T$. maya presenta un sensibile allungamento delle appendici, elitre saldate, atterismo ed occhi ridotti a pochi ommatidi completamente privi di pigmento.

Grazie alla cortesia del Dr. R.L. Wenzel del Field Museum of Natural History di Chicago ho potuto avere in studio altri Histeridae del Nuovo Mondo tutti provenienti da grotte messicane. Tra questi interessantissimi esemplari ho potuto isolare altre tre nuove specie appartenenti a Troglobacanius.

In totale sono per ora note quattro specie di questo genere troglobio che sembra essere molto diffuso nelle grotte della Sierra Madre Oriental messicana:

- T. maya Vomero, Gruta de Coconà, Tabasco.

- T. reddelli Vomero, Grutas de el Puente e Cueva de los Vampiros, Sierra del Guatemala, Tamaulipas.

- T. bolivari Vomero, Sotano del Tigre, Sierra de El Abra, San Louis Potosi.

- T. sbordonii Vomero, Sotano de Gomez Farias, Sierra del Guatemala, Tamaulipas.

Le quattro specie sono state raggruppate in due gruppi di specie; il gruppo maya riunisce le tre specie di dimensioni minori che sono caratterizzate da una simile struttura dell'edeago e della spermateca (maya, reddelli e bolivari); il gruppo maya popola sia regioni più settentrionali (Sierra de El Abra e Sierra del Guatemala) sia regioni più meridionali (Tabasco). Il gruppo sbordonii è noto soltanto con la specie nominale che popola una grotta della Sierra del Guatemala vicina geograficamente ad una grotta popolata da $T$. reddelli del gruppo maya. (per più esaurienti notizie sulla filogenesi e sulla speciazione di Troglobacanius vedi Vomero 1973).

L'importanza della scoperta di questo genere è notevole in primo luogo poichè si tratta del primo genere di Histeridae troglobio descritto del Nuovo Mondo e di uno dei pochi Coleotteri troglobi delle grotte delle regioni tropicali; e poi perchè ripropone ancora una volta il problema del popolamento delle grotte tropicali, argomento trattato in questo stesso Congresso.

Da un accurato studio del materiale messicano, soprattutto in base a caratteri dei genitali, fino ad ora scarsamente utilizzati nella sistematica degli Histeridae, Troglobacanius risulta appartenere alla linea filetica di "Bacanius". Poco si può dire però sulle reali affinità del genere prima di una revisione delle specie del gruppo Bacanius. La sistematica di questo gruppo di generi è in corso di completa revisione. Da preliminari osservazioni da me effettuate Bacanius va considerato un genere polifiletico.

Geoculus caecus Wenzel 1944

Quest'ultimo genere cieco a brachittero, descritto degli Stati Uniti (Georgia), non mostra nessuna connessione con fenomeni carsici. E' stato raccolto nel corso di una ricerca sulla fauna del suolo di un frutteto. 
E' nettamente distinto dal gruppo Bacanius mentre sembra presentare problematiche affinità con il genere Triballodes.

\section{HISTERIDI TROGLOFILI E GUANOBI}

La presenza in grotta di Histeridae a costumi troglofili o guanobi è maggiore del numero delle specie troglobie. La letteratura riporta un certo numero di specie provenienti da varie cavità delle più svariate regioni, ma queste citazioni sono da prendersi con cautela a causa delle determinazioni a volte grossolanamente errate. Le uniche determinazioni sicure si riferiscono a Tribalus cavernicola conosciuto di alcune grotte dell'Africa orientale, e ad alcune specie di Gnatoncus presenti in grotte paleartiche.

A queste scarse notizie occorre aggiungere alcune segnalazioni originali riguardanti materiale proveniente da grotte messicane a carattere tropicale. Anche quest'ultimo lotto di Histeridae è frutto delle recenti ricerche biospeleologiche condotte dall'Istituto di Zoologia dell'Università di Roma in Messico. Tra il materiale più interessante è da segnalare la presenza di una nuova specie appartenente ad un nuovo genere affine a Bacanius, la cui descrizione verrà pubblicata quanto prima; di numerosi esemplari di Euspilotus (Neosaprinus) scrupularis Le Conte, di una nuova specie di $E$. (Neosaprinus), e di una nuova specie di Operclipygus vicina ad $O$. hamistrius.

Nelle grotte tropicali messicane, dove molto probabilmente fanno parte della biocenosi guanobia, gli Histeridae sono presenti in percentuale relativamente alta e per numero di specie e, soprattutto, per numero di individui, in rapporto alle altre famiglie di Coleotteri.

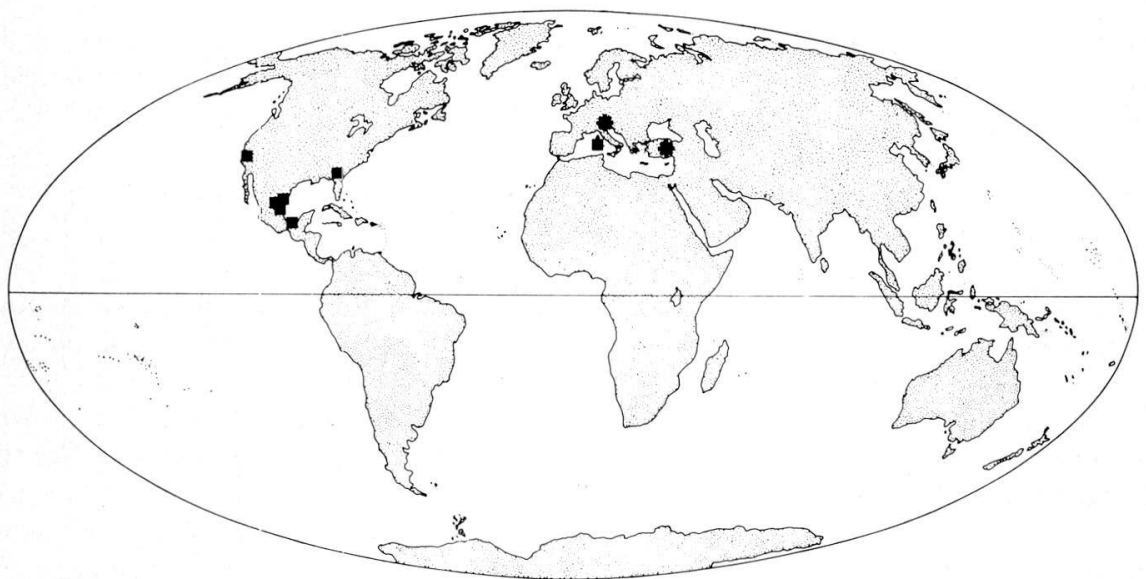

Distribuzione mondiale degli Histeridae troglobi ed edafobi. $($ Quadrati $=$ Dendrophilinae del gruppo Bacanius; aste $:$ ischi = Abraeinae. ) 


\section{CONCLUSIONI}

Per concludere, per quanto ci è dato di conoscere, anche se le ricerche in molte regioni non sono ancora ad un livello sufficiente (almeno per le regioni tropicali), la colonizzazione da parte degli Histeridae delle grotte e, in senso più lato, di tutti gli ambienti ipogei, si è attuata in modesta misura.

Si può osservare che nelle grotte tropicali gli Histeridae fanno abitualmente parte della comunità cavernicola troglobia e guanobia dove sono presenti, a volte, in notevole numero di individui e dove svolgono un preciso ruolo nella catena trofica dell'ecosistema, mentre nelle grotte temperate, a parte i rarissimi troglobi, sono molto poco rappresentati. D'altro canto, a causa della scarsità dei reperti, non è ancora chiaro in che misura gli Histeridae "troglobi" delie grotte temperate facciano realmente parte della comunità di grotta.

Nelle specie descritte come troglobie l'analisi morfologica non fornisce elementi sufficienti per valutare il grado d'adattamento all'ambiente speleo.

Probabilmente una risposta a questo quesito potrà essere data soltanto da allevamenti che permettano di valutare il grado di specializzazione raggiunto da questi animali a livello fisiologico.

\section{RIASSUNTO}

Nella presente comunicazione vengono fatte alcune considerazioni sugli Histeridae troglobi ed edafobi della fauna mondiale. Specie per specie vengono trattati tutti gli Histeridae spettanti alla fauna sotteranea che presentano assenza o estrema riduzione degli occhi. Le specie considerate appartengono ai generi Speleacritus Jeannel, Spelaeabraeus Moro, Sardulus Patrizi, Bacanius Le Conte, Troglobacanius Vomero e Geoculus Wenzel. Vengono infine fatte alcune considerazioni sugli Histeridae troglofili e guanobi, segnalando la presenza di un nuovo genere e di alcune nuove specie spettanti alla biocenosi guanobia delle grotte tropicali messicane.

\section{SUMMARY}

The author makes some considerations on troglobitic and endogeous world Histeridae. All these species present extreme reduction of eyes or are completely blind; all are wingless and only one is brachipterous.

The Histeridae described here belong to the following genera: Spelacritus Jeannel and Spelaeabraeus Moro (Abraeinae), Sardulus Patrizi, Bacanius Le Conte, Troglobacanius Vomero and Geoculus Wenzel. (Dendrophilinae, Bacanius group).

Finally some considerations on troglophilic and guanobitic Histeridae are made, reporting the recent discovery of a new genus and of some new species belonging to guanobitic biocenosis of Mexican tropical caves. 


\section{BIBLIOGRAFIA}

BUSULINI, E., 1958. Nota preliminare su alcune entità cavernicole nuove o poco note della regione veneta. - Boll. Mus. Civ. Storia Nat. Venezia, XI: 11-17.

CASEY, T.L., 1893. Coleopterological Notices V. - Ann. N.Y. Acad. Sci., 7: 553-578.

JEANNEL, R., 1934. Coleopteres cavernicoles de la grotte de Fersine, en Asie Mineure. - Ann. Soc. Ent. France, CIII: 159-174.

MORO, G.B., 1957. Una nuova specie italiana di Histeridae cieco appartenente ad un nuovo genere. - Boll. Soc. Ent. It., LXXXII (1-2): 9-13.

PATRIZI, S., 1955. Surdulus spelaeus n. gen. n. sp. - Fragmenta Entomologica, II (6) 9-13.

ROSSI, C., 1967. Una nuova specie di Spelaeabraeus delle Prealpi Carniche (Col. Histeridae). - Boll. Soc. Ent. It., XCVII (5-6): 89-93.

VOMERO, V., 1973. Troglobacanius n. gen. with four new species, a line of cave adapted Histeridae. - In Subterranean Fauna of Mexico II. Quaderni Accad. Naz. Lincei, 171 (in corso di stampa).

WENZEL, R.L., 1944. On the classification of the Histerid beetles. - Field Mus. Nat. Hist., Zool. Ser. 28 (2): 51-151. 